\title{
Reduced resolution depth coding for stereoscopic 3D video
}

\begin{abstract}
In this paper, Reduced Resolution Depth Compression (RRDC) is proposed for Scalable Video Coding (SVC) to improve the 3D video rate distortion performance. RRDC is applied by using Down-Sampling and Up-Sampling (DSUS) of the depth data of the stereoscopic 3D video. The depth data is down-sampled before SVC encoding and up-sampled after SVC decoding operation. The proposed DSUS method reduces the overall bit rates and consequently: 1) improves SVC rate distortion for 3D video, particularly at lower bit rates in error free channels; and 2) improves 3D SVC performance for 3D transmission in error prone channels. The objective quality evaluation of the stereoscopic 3D video yields higher PSNR values at low bit rates for SVCDSUS compared to the original SVC (SVC-Org), which makes it advantageous in terms of reduced storage and bandwidth requirements. Moreover, the subjective quality evaluation of the stereoscopic 3D video further confirmed that the perceived stereoscopic 3D video quality of the SVC-DSUS is very similar to the stereoscopic 3D video of the SVC-Org by up to $98.2 \%$.
\end{abstract}

Keyword: Image resolution; Rate distortion theory; Stereo image processing; Video coding 